\title{
SELF-DUAL SU(5) MONOPOLE SOLUTIONS
}

\author{
Carl L. GARDNER \\ Department of Physics, Bowdoin College, Brunswick, ME 04011, USA
}

Received 23 March 1984

\begin{abstract}
The static spherically symmetric self-dual SU(5) monopole solutions which correspond to stable monopoles outside of the Prasad-Sommerfield limit are constructed. These are the monopole solutions appropriate for perturbative calculations (of monopole masses, stability, proton decay and weak interaction catalysis, fermion-monopole bound states, etc.) about the PS limit. The symmetries of the monopoles as $r \rightarrow 0$ are also discussed.
\end{abstract}

1. Introduction. In the $\mathrm{SU}(5)$ grand unified theory, magnetic monopoles with magnetic charges (in units of the Dirac charge $\left.1 / 2 e^{\neq 1}\right) 1$ (single monopole), 2 (double), 3 (triple), 4 (quadruple), and 6 (sextuple) are stable if the superheavy Higgs particles are lighter than the superheavy vector bosons and if the singlet Higgs particle is lighter than the octet and triplet Higgs particles [1]. In this letter, I will consider the symmetry breaking $\mathrm{SU}(5) \rightarrow \mathrm{SU}(3) \times \mathrm{SU}(2) \times \mathrm{U}(1)$ and construct the static spherically symmetric self-dual monopole solutions which correspond to stable monopoles when the self-duality restriction is relaxed.

The magnetic field of the monopole $B=Q / 2 g r^{2}$ asymptotically on the positive $z$ axis, where $Q$ is a linear combination of unbroken generators. In unitary gauge, the upper $3 \times 3$ corner of $Q$ represents $S U(3)$, the lower $2 \times 2$ corner represents $S U(2)$, while $U(1)$ is represented along the diagonal. Since $Q$ is hermitian, $Q$ can be diagonalized along the positive $z$ axis by an $\mathrm{SU}(3) \times \mathrm{SU}(2) \times \mathrm{U}(1)$ gauge transformation. In this gauge, $Q$ is constructed out of the generators $Y$ $=\operatorname{diag}(1 / 3,1 / 3,1 / 3,-1 / 2,-1 / 2), Y_{8}=\operatorname{diag}(1 / 3,1 / 3$, $-2 / 3,0,0)$, and $t_{3}=\operatorname{diag}(0,0,0,1 / 2,-1 / 2)$. The solution of the quantization and Brandt-Neri [2] stability conditions is $Q=n Y+n_{8} Y_{8}+n_{3} t_{3}$, where $n$ is the integral magnetic charge in Dirac units, and $n_{8}=0, \pm 1$ and $n_{3}=0,-1(+1$ and -1 are SU(2)-gauge equivalent)

${ }^{\ddagger 1}$ The electronic charge $e=(3 / 8)^{1 / 2} g$, where $g$ is the SU(5) coupling constant. are uniquely determined by the quantization requirement that each eigenvalue of $Q$ be an integer. The double, triple, quadruple, and sextupel monopoles, in addi. tion to having stable magnetic fields, are stable against decay into lighter monopoles if the Higgs particles are lighter than $M_{\mathrm{X}}$ and if the singlet Higgs particle is lighter than the triplet and octet Higgs particles [1] .

The spherically symmetric quadruple monopoles are unstable against the emission of gluons or W's [1] Thus only the single, double, triple, and sextuple monopole solutions will be constructed in this letter.

In the Prasad-Sommerfield (PS) limit, the BrandtNeri stability condition is not applicable. The monopoles with Dirac charge $n>1$ are neutrally stable against decay into $n$ single monopoles. However for perturbative calculations (of monopole masses, stability, proton decay and weak interaction catalysis, fermion-monopole bound states, etc.) about the PS limit, only the solutions which correspond to stable monopoles outside of this limit are of interest.

In the PS limit, the monopole fields satisfy the selfduality or Bogomol'nyi equations $B_{i}=D_{i} \phi[3]$ (in the $A_{0}=0$ gauge), where

$B_{i}=\frac{1}{2} \epsilon_{i j k} F_{j k}, \quad F_{i j}=\partial_{i} A_{j}-\partial_{j} A_{i}+\mathrm{i} g\left[A_{j}, A_{k}\right]$,

$D_{i} \phi=\partial_{i} \phi+\mathrm{i} g\left[A_{i}, \phi\right]$.

The boundary conditions at infinity follow from requiring that $\phi$ and $A_{i}$ approach their vacuum values at infinity: $\phi \rightarrow v \operatorname{diag}(1,1,1,-3 / 2,-3 / 2)^{\neq 2}$ and $A_{i} \rightarrow 0$. 
In addition, the asymptotic form of $B$ imposes the boundary condition that $\hat{r} \cdot B=\mathrm{d} \phi / \mathrm{d} r=Q / 2 g r^{2}$ asymptotically. To guarantee finiteness of the monopole mass, I will require $\phi \rightarrow$ const. as $r \rightarrow 0$ and $A_{i} \rightarrow 0$ as $r$ $\rightarrow 0$. In constructing the self-duel solutions, I will follow the methods of Wilkinson and Goldhaber [4] and Leznov and Saveliev [5]. Similar mentods have been employed by Bose and McGlinn [6] in constructing different self-dual SU(5) solutions. Only the single and sextuple (maximal SU(2) embedding) solutions of these authors are stable outside of the PS limit.

Wilkinson and Bais [7] constructed the maximal-embedding solution implicitly for $\mathrm{SU}(n)$, while Bose and McGlinn independently found the same explicit sextuple solution for SU(5) that I found.

The monopole solutions are spherically symmetric with respect to $J=L+T$, where $T$ classifies an SU(2) embedding in SU(5). The general definition of spherical symmetry in a gauge theory is that a rotation of the coordinate system can be compensated for by a local gauge transformation. In the case of finite-energy monopole solutions, the local gauge transformation can always be replaced by a global SU(2) transformation $T$ [8].

The monopole fields may be expressed in radial gauge as

$A_{i}=g^{-1} \epsilon_{i j k} \hat{r}_{j}\left(N_{k}-T_{k} / r\right), \phi=g^{-1} \Phi$,

where $N=N(r, \hat{r})$ and $\Phi=\Phi(r, \hat{r})$. The condition of spherical symmetry implies $\left[J_{i}, \phi\right]=0$ and $\left[J_{i}, A_{j}\right]$ $=\mathrm{i} \epsilon_{i j k} A_{k}$. Henceforth, all fields will be expressed on the positive $z$ axis. Fields at any other point may be constructed by spherical symmetry. Set $\Phi=\Psi-T_{3} / r$ and $N_{ \pm}=N_{1} \pm \mathrm{i} N_{2}$. The Bogomol'nyi equations take the form

$\Psi^{\prime}=-\frac{1}{2}\left[N_{+}, N_{-}\right], N_{ \pm}^{\prime}=\mp\left[\Psi, N_{ \pm}\right]$.

The constraints due to spherical symmetry reduce to $\left[T_{3}, \Psi\right]=0$ and $\left[T_{3}, N_{ \pm}\right]= \pm N_{ \pm} . N_{-}$may be set equal to $\left(N_{+}\right)^{t}$. For each $T_{3}$ embedding, the constraint $\left[T_{3}, N_{+}\right]=N_{+}$sets most of the elements of $N_{+}$equal to zero. Label the nonzero entries $\alpha_{i}$. Then for each embedding $T_{3}$, the Bogomol'nyi equations can be cast in the form

\footnotetext{
\#2 $\mathrm{g} v$ defines a fundamental mass in the SU(5) problem, and
} will henceforth be set $=1$. In these units, $M_{\mathrm{X}}=5 \mathrm{gv} / 2=5 / 2$. $\psi_{i}^{\prime}=-\frac{1}{2} \alpha_{i}^{2}, \quad \alpha_{i}^{\prime}=-\sum_{j} K_{i j} \psi_{j} \alpha_{i}$,

where $\psi_{i}$ is defined below for each $T_{3}$ embedding, and where $K_{i j}$ is a symmetrical matrix with 2's along the diagonal and either -1 or 0 off the diagonal. Next define $\theta_{j}$ by $\ln \left(\alpha_{i}^{2}\right)=\Sigma_{j} K_{i j} \theta_{j}$. Then $\theta_{j}^{\prime}=-2 \psi_{j}$ and the Bogomol'nyi equations are transformed to

$\theta_{j}^{\prime \prime}=\exp \left(\sum_{k} K_{j k} \theta_{k}\right)$.

The boundary conditions for $\theta$ are determined from the boundary conditions for $\Phi$. These equations will be solved below for $\Phi$ for the single, double, triple, and sextuple monopoles. Asymptotically $B=B \hat{r}$. In each case, $B=\mathrm{d} \phi / \mathrm{d} r$ will be calculated. $A$ can be found from eq. (5).

2. Single monopole. The single monopole is described by $T_{3}=\operatorname{diag}(0,0,1 / 2,-1 / 2,0)$. The constraint $\left[T_{3}, N_{+}\right]=N_{+}$implies that

$N_{+}=\left[\begin{array}{lllll}0 & 0 & 0 & 0 & 0 \\ 0 & 0 & 0 & 0 & 0 \\ 0 & 0 & 0 & \alpha & 0 \\ 0 & 0 & 0 & 0 & 0 \\ 0 & 0 & 0 & 0 & 0\end{array}\right]$.

Set $\Psi=\operatorname{diag}(1,1, \psi-1 / 4,-\psi-1 / 4,-3 / 2)$.

$\Phi$ is determined from the Bogomol'nyi equations:

$\Phi=\operatorname{diag}\left(1,1, \Phi_{0}-1 / 4,-\Phi_{0}-1 / 4,-3 / 2\right)$,

$B=\operatorname{diag}(0,0,1,-1,0) / 2 g r^{2}$,

where $\Phi_{0}=\frac{5}{4} \operatorname{coth} \frac{5}{2} r-1 / 2 r$. If dimensional units are put back in, $\Phi_{0}=\frac{1}{2} M_{\mathrm{X}} \operatorname{coth} M_{\mathrm{X}} r-1 / 2 r$.

3. Double monopole. Consider two single monopoles with $Q=\operatorname{diag}(0,1,0,-1,0)$ and $Q^{\prime}=\operatorname{diag}(1,0$, $0,0,-1)$. In this gauge orientation, the repulsive exchange of $U(1)$ vector particles is exactly cancelled by the attractive $W$ and gluon exchange, and the attractive exchange of scalar Higgs particles is exactly cancelled by repulsive non-abelian Higgs exchange [1]. Thus in this gauge, two single monopoles may be brought together without interaction in different SU(2) subgroups to form a double monopole.

Such a classical solution may be constructed by taking $T_{3}=\operatorname{diag}(1 / 2,1 / 2,0,-1 / 2,-1 / 2) . N_{+}$may be taken equal to 
$N_{+}=\left[\begin{array}{lllll}0 & 0 & 0 & \alpha & 0 \\ 0 & 0 & 0 & 0 & \alpha \\ 0 & 0 & 0 & 0 & 0 \\ 0 & 0 & 0 & 0 & 0 \\ 0 & 0 & 0 & 0 & 0\end{array}\right]$.

Set $\Psi=\operatorname{diag}(\psi-1 / 4, \psi-1 / 4,1,-\psi-1 / 4,-\psi-1 / 4)$. Then $\Phi$ is found from the Bogomol'nyi equations:

$\Phi=\left(\Phi_{0}-1 / 4, \Phi_{0}-1 / 4,1,-\Phi_{0}-1 / 4,-\Phi_{0}-1 / 4\right)$,

$B=\operatorname{diag}(1,1,0,-1,-1) / 2 g r^{2}$.

4. Triple monopole. Choose $T_{3}=\operatorname{diag}(1,1 / 2,0$, $-1 / 2,-1)$. Then

$N_{+}=\left[\begin{array}{lllll}0 & 0 & \alpha_{1} & 0 & 0 \\ 0 & 0 & 0 & \alpha_{2} & 0 \\ 0 & 0 & 0 & 0 & \alpha_{3} \\ 0 & 0 & 0 & 0 & 0 \\ 0 & 0 & 0 & 0 & 0\end{array}\right]$.

Set $\Psi=\operatorname{diag}\left(\psi_{1}, \psi_{2}, \psi_{3}-\psi_{1},-\psi_{2},-\psi_{3}\right)+\operatorname{diag}(1 / 6$, $-1 / 4,1 / 6,-1 / 4,1 / 6)$. The solution is described most simply in terms of $\exp \left(-\theta_{i}\right)$ :

$\exp \left(-\theta_{1}\right)=\frac{1}{5}\left[\left(r-\frac{1}{5}\right) \exp \left(\frac{5}{3} r\right)+\frac{1}{5} \exp \left(-\frac{10}{3} r\right)\right]$,

$\exp \left(-\theta_{2}\right)=\frac{2}{25} \sinh \frac{5}{2} r$,

$\exp \left(-\theta_{3}\right)=\exp \left[-\theta_{1}(-r)\right]$

$B=\operatorname{diag}(1,1,1,-1,-2) / 2 g r^{2}$.

5. Sextuple monopole. The stable sextuple monopole corresponds to the maximal embedding $T_{3}$

$=\operatorname{diag}(2,1,0,-1,-2)$. The constraint of spherical symmetry implies

$N_{+}=\left[\begin{array}{lllll}0 & \alpha_{1} & 0 & 0 & 0 \\ 0 & 0 & \alpha_{2} & 0 & 0 \\ 0 & 0 & 0 & \alpha_{3} & 0 \\ 0 & 0 & 0 & 0 & \alpha_{4} \\ 0 & 0 & 0 & 0 & 0\end{array}\right]$.

The ansatz for $\Psi$ is $\Psi=\operatorname{diag}\left(\psi_{1}, \psi_{2}-\psi_{1}, \psi_{3}-\psi_{2}\right.$, $\left.\psi_{4}-\psi_{3},-\psi_{4}\right)$. The solution to the Bogomol'nyi equations is

$$
\begin{aligned}
& \exp \left(-\theta_{1}\right)=\frac{1}{25}\left[\left(\frac{1}{2} r^{2}-\frac{2}{5} r+\frac{3}{25}\right) \exp (2 r)\right. \\
& \left.\quad-\left(\frac{1}{5} r+\frac{3}{25}\right) \exp (-3 r)\right], \\
& \exp \left(-\theta_{2}\right)=\frac{1}{125}\left[\left(\frac{1}{10} r^{2}-\frac{2}{25} r+\frac{1}{125}\right) \exp (4 r)\right. \\
& \left.\quad+\left(\frac{1}{2} r^{3}+\frac{1}{10} r^{2}+\frac{2}{25} r-\frac{2}{125}\right) \exp (-r)+\frac{1}{125} \exp (-6 r)\right], \\
& \exp \left(-\theta_{3}\right)=\exp \left[-\theta_{2}(-r)\right], \\
& \exp \left(-\theta_{4}\right)=\exp \left[-\theta_{1}(-r)\right],
\end{aligned}
$$$$
B=\operatorname{diag}(2,2,2,-3,-3) / 2 g r^{2} \text {. }
$$

6. Symmetries at short distances. In the 't HooftPolyakov [9] monopole solution, $\phi \rightarrow 0$ as $r \rightarrow 0$ and thus the gauge symmetry at short distances is the full $\mathrm{SU}(2)$ group. For monopoles in larger gauge groups, new unbroken symmetries exist inside the monopole core ( $r \ll M$, where $M$ is the mass of the heavy vector gauge particles). In table 1 , the unbroken symmetries at short distances for the stable SU(5) monopoles are presented. The full SU(5) symmetry is restored at short distances only for the sextuple monopole. The new unbroken symmetries may influence monopole

Table 1

Monopole symmetries at short distances.

\begin{tabular}{llll}
\hline Monopole & $\Phi$ as $r \rightarrow 0$ & Unbroken symmetry \\
\hline single & $\operatorname{diag}(1,1,-1 / 4,-1 / 4,-3 / 2)$ & $\mathrm{SU}(2) \times \mathrm{SU}(2) \times \mathrm{U}(1)$ \\
double & $\operatorname{diag}(-1 / 4,-1 / 4,1,-1 / 4,-1 / 4)$ & $\mathrm{SU}(4) \times \mathrm{U}(1)$ \\
triple & $\operatorname{diag}(1 / 6,-1 / 4,1 / 6,-1 / 4,1 / 6)$ & $\mathrm{SU}(3) \times \mathrm{SU}(2) \times \mathrm{U}(1)$ \\
& sextuple & $\operatorname{diag}(0,0,0,0,0)$ & $\mathrm{SU}(5)$ \\
\hline
\end{tabular}


catalysis of nucleon decay and weak interactions, the gauge hierarchy problem [10] in the presence of a monopole, and monopole-fermion bound states.

My thanks are due to Alan Guth, Jeffrey Harvey, Nick Manton, and Henry Tye for valuable discussions. I am also grateful for the opportunity of attending the Seattle Institute on Phase Transitions in Gauge Theories at the University of Washington, where part of this work was completed.

\section{References}

[1] C.L. Gardner and J.A. Harvey, Phys. Rev. Lett. 52 (1984) 879.
[2] R.A. Brandt and F. Neri, Nucl. Phys. B161 (1979) 253.

[3] E.B. Bogomol'nyi, Sov. J. Nucl. Phys. 24 (1976) 449.

[4] D. Wilkinson and A.S. Goldhaber, Phys. Rev. D16 (1977) 1221.

[5] A.N. Leznov and M.V. Saveliev, Lett. Math. Phys. 3 (1979) 489; Commun. Math. Phys. 74 (1980) 111.

[6] D. Wilkinson and F.A. Bais, Phys. Rev. D19 (1979) 2410.

[7] S.K. Bose, University of Notre Dame preprint; S.K. Bose and W.D. McGlinn, University of Notre Dame preprint.

[8] R. Jackiw and N.S. Manton, Ann. Phys. (NY) 127 (1980) 257 ;

R. Jackiw, Acta Physica Austriaca Suppl. XXII (1980) 383;

E.J. Weinberg, Phys. Lett. 136B (1984) 179.

[9] G. 't Hooft, Nucl. Phys. B79 (1974) 276;

A.M. Polyakov, JETP Lett. 20 (1974) 194.

[10] J.A. Harvey and E. Witten, unpublished. 